\title{
Ischemic Stroke as a Complication of Chemotherapy in a Child with Acute Lymphoblastic Leukemia-Case Report
}

Joanna Zawitkowska ${ }^{1^{*}}$, Monika Lejman ${ }^{1,2}$

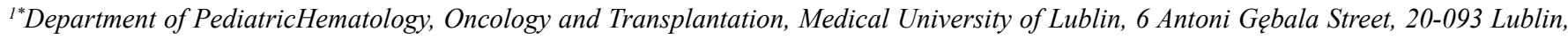
Poland.

${ }^{2}$ Department of PediatricHematology, Oncology and Transplantation, Medical University of Lublin, University Children's Hospital, Genetic Diagnostic Laboratory, 6 Antoni Gębala Street, 20-093 Lublin, Poland

\author{
Article Details \\ Article Type: Case Report \\ Received date: $26^{\text {th }}$ February, 2020 \\ Accepted date: $30^{\text {th }}$ March, 2020 \\ Published date: $01^{\text {st }}$ April, 2020
}

"Corresponding Author: Joanna Zawitkowska, Department of PediatricHematology, Oncology and Transplantation, Medical University of Lublin, 6 Antoni Gębala Street, 20-093 Lublin, Poland. E-mail: jzawitkowska1971@gmail.com

Citation: Zawitkowska, J., Lejman, M. (2020). Ischemic stroke as a complication of chemotherapy in a child with acute lymphoblastic leukemia - case report. J Case Reports Cancer 1(1):101. doi: https://doi.org/10.33790/jcrc1100101.

Copyright: (C2020, This is an open-access article distributed under the terms of the Creative Commons Attribution License 4.0, which permits unrestricted use, distribution, and reproduction in any medium, provided the original author and source are credited.

\section{Abstract}

Central and/or peripheral neuropathy are common and serious disorder during chemotherapy in children with acute lymphoblastic leukemia. We report a patient with acute lymphoblastic leukemia (ALL) and neurological complications, in whom the treatment were a difficult challenge.Medical records of this child were reviewed for demographic, clinical, and laboratory data. Patients were hospitalized in the Department of Pediatric Hematology, Oncology and Transplantology of the Medical University of Lublin, Poland. The child was diagnosed with B-cell precursor common positive ALL and chemotherapy was started in January 2019. She was classified as intermediate risk group. On day 29 of this phase, her condition worsened: at first contact with the child was difficult, she could not speak, move, and then convulsions of the left half of the body appeared. Laboratory data presented hyponatremia $(131 \mathrm{mmol} / \mathrm{l})$ and increased CRP (C-reactive protein; $1.43 \mathrm{mg} / \mathrm{dl})$. Chemotherapy was interrupted. Magnetic resonance imaging (MRI) of central nervous system (CNS) shows the extensive ischemic stroke in the left temporal lobe and thrombosis in the sigmoid and transverse sinus of the brain. She received low molecular weight heparin subcutaneously, intravenous anticonvulsant (phenobarbital), the broad-spectrum antibiotic and antifungal drug and parenteral nutrition. Her general condition gradually improved. After 2 weeks of therapy, MRI of CNS presents the partial regression of ischemic changes and complete one of thrombosis.After 4 weeks, all neurological symptoms withdrew and continued chemotherapy (without vincristine). The child completed the reinduction phase of therapy in October 2019 and started maintenance chemotherapy 2 weeks later. Despite anticonvulsant drug, convulsions of the left half of the body occurred in November 2019 and January 2020. In conclusion, it is important to an analysis and discussion of supportive care to reduce these toxicities in children with ALL.

Key words: Child, Acute Lymphoblastic Leukemia, Neurologic Complications, Ischemic Stroke, Thrombosis

\section{Introduction}

Currently, over $80 \%$ children with acute lymphoblastic leukemia are cured. This is due to application of intensive chemotherapy, while on the other hand it brings the riskof several other health complications $[1,2]$. Central and/or peripheral neuropathy are infrequent, but serious adverse events. This may lead to deviations in chemotherapy, impact on the treatment outcome and patients' quality of life. Thus, it is important to improve supportive therapy for these patients [3].

We report a patient with acute lymphoblastic leukemia (ALL) and neurological complications, in whom the treatment were a difficult challenge.Medical records of this child were reviewed for demographic, clinical, and laboratory data. Patients were hospitalized in the Department of PediatricHematology, Oncology and Transplantology of the Medical University of Lublin, Poland.

\section{Case report}

A 3-year old girl was admitted to our hospital because of fever, weakness, vomiting, nausea and abdominal pain. There were no comorbidities as well as no significant aspects of the patient's family history.

The child was diagnosed with B-cell precursor common positive ALL and chemotherapy was started in January 2019, according to AIEOP-BFM 2017 (International collaborative treatment protocol for childrenand adolescents with acute lymphoblastic leukemia) [4]. She was classified as intermediate risk group (genetics results: BCR/ABL1 - negative, KMT2A - negative, ETV6/RUNX1 positive,TCF3 - negative, karyotype - normal, IKZF1 - negativea good response to steroids: on day 8 blast count in peripheral blood $<1000 / \mu 1$; myelogram on day 15: $0 \%$ blasts and minimal residual disease by flow cytometry (MRD): $3.9 \%$; myelogram on day 33 : $2.4 \%$ blasts and MRD by PCR method (polymerase chain reaction): $<1 \times 10-4)$. Leukemic infiltration of central nervous system (CNS) was no observed at the time the diagnosis. This was excluded in tests such as an analysis of cerebrospinal fluid, neurological tests and EEG (electroencephalography). She received the induction (Protocol IA-Pred) and consolidation (Protocol M) phase of therapy without severe complications. The reinduction therapy (protocol II) was well tolerated to day 28 th. On day 29 of this phase, her condition worsened: at first contact with the child was difficult, she could not speak, move, and then convulsions of the left half of the body appeared. Laboratory data presented hyponatremia $(131 \mathrm{mmol} / \mathrm{l})$ and increased CRP (C-reactive protein; $1.43 \mathrm{mg} / \mathrm{dl}$ ). No leukopenia and neutropenia have been observed at this time.Chemotherapy was interrupted. Magnetic resonance imaging (MRI) of CNS shows the extensive ischemic stroke in the left temporal lobe and thrombosis in the sigmoid and transverse sinus of the brain (Picture 1). She received low molecular weight heparin subcutaneously, intravenous 
anticonvulsant (phenobarbital), the broad-spectrum antibiotic (cefepim, amikacin) and antifungal drug (micafungin) and parenteral nutrition. Her general condition gradually improved. The girl was rehabilitated. After 2 weeks of therapy, MRI of CNS presents the partial regression of ischemic changes and complete one of thrombosis.After 4 weeks, all neurological symptoms withdrew and continued chemotherapy (29 day of the reinduction phase of therapy). However, due to neurological complication a dose of vincristine was omitted. She received only daunorubicin. She still continues anticonvulsant (oral phenobarbital) and low molecular weight heparin subcutaneously. The child completed the reinduction phase of therapy in October 2019 and started maintenance chemotherapy 2 weeks later. Despite anticonvulsant drug, convulsionsof the left half of the body occurred in November 2019. Levetiracetam was applied instead of phenobarbital. MRI of CNS present no new ischemic changes. The next 2 month, convulsions once again and a dose of levetiracetam was increase. Currently, she is alive and well.

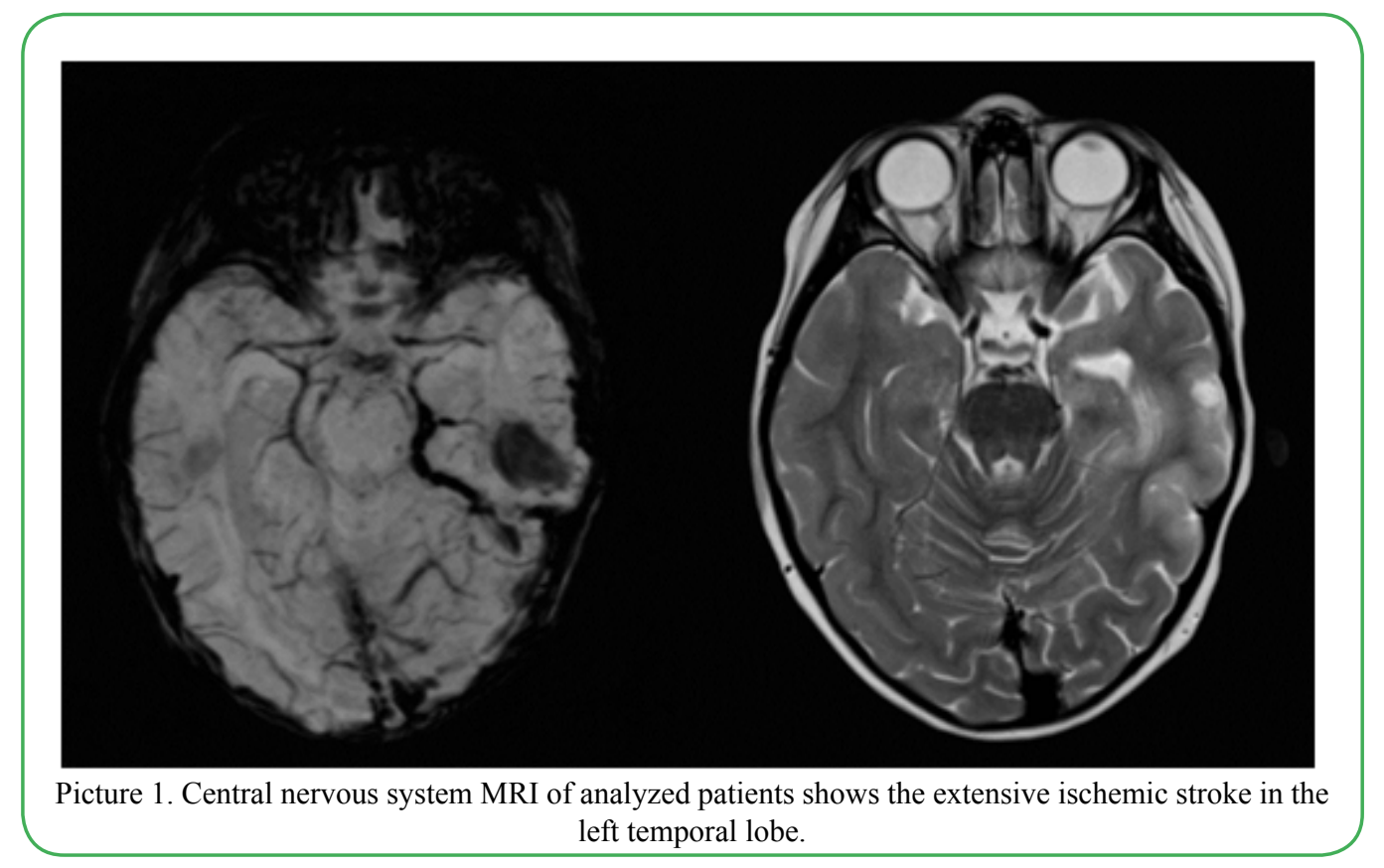

\section{Discussion}

Complications related to therapy reduce the cure rate of childhood acute lymphoblastic leukemia by $2-3 \%$ of deaths as a result of toxicity [5]. Neurological complications are serious therapeutic problem in children undergoing chemotherapy. The central nervous system (CNS) toxicity during chemotherapy occur in $10-15 \%$ of all children in childhood. Peripheral neuropathies are common, usually caused by vincristine, and are generally completely reversible, but improvement may take several months [3]. Nazir et al. published that 19 out of $103(1.8 \%)$ children developed VCR-related neuropathy. Polyneuropathy appeared after 2-11 doses of VCR [6].

In another study, authors present that neurotoxicity was diagnosed in 49/1379 (3.6\%) patients and diagnosed during the induction phase of therapy in $55 \%$ of cases[1].

Baytan et al. published a retrospective analysis of 323ALL cases. Twenty-three of these children (7.1\%) experienced central nervous system side effects during acute lymphoblastic leukemia therapy. Similar to previous author, the majority of those complications developed during induction [7].

Our large,retrospective study demonstrated that neurotoxic events both peripheral and central accounted for $2.9 \%$ and were observed during induction and reinduction phases of treatment [8]. In the analyzedpatient, neurotoxic events occurred during intensive therapy, which included steroids (dexamethosone), vincristine and PEG-asparaginase. Probably, these cytostatics are responsible for intense neurotoxicity. This is why, it is important to an analysis and discussion of supportive care to reduce these toxicities in children with ALL.

\section{Formatting of funding sources:}

This research did not receive any specific grant from funding agencies in the public, commercial, or not-for-profit sectors.

Conflict of interest: The authors have declared no conflict of interest.

\section{Patient(s)/Participant(s) Consent Statement: signed.} References

1. Millan, N.C., Pastrana, A., Guitter, M.R., Zubizarreta, P.A., Monges, M.S., \& Felice, M.S. (2018). Acute and sub-acute neurological toxicity in children treated for acute lymphoblastic leukemia. Leuk Res 65:86-93.

2. Wolthers, B.O., Frandsen, T.L., Baruchel, A., Attarbaschi, A., Barzilai, S., \& Colombini, A... (2017). Asparaginase-associated pancreatitis in childhood acute lymphoblastic leukaemia: an observational Ponte di Legno Toxicity Working Group study. Lancet Oncol 18(9):1238-48.

3. Schmiegelow, K., Muller, K., Mogensen, S.S., Mogensen, P.R., Wolthers, B.O., \& Stoltze, U.K... (2018). Non-infectious chemotherapy-associated acute toxicities during childhood acute lymphoblastic leukemia therapy. F1000Res 6:444.

4. Stanulla, M., Dagdan, E., Zaliova, M., Moricke, A., Palmi, C., Cazzaniga, G... (2018). IKZF1(plus) Defines a New Minimal Residual Disease-Dependent Very-Poor Prognostic Profile in Pediatric B-Cell Precursor Acute Lymphoblastic Leukemia. J Clin Oncol 36(12):1240-9.

5. Stary, J., Zimmermann, M., Campbell, M., Castillo, L., Dibar, E., Donska, S... (2014). Intensive chemotherapy for childhood acute lymphoblastic leukemia: results of the randomized intercontinental trial ALL IC-BFM 2002. J Clin Oncol 32(3):174-84.

6. Nazir, H.F., AlFutaisi, A., Zacharia, M., Elshinawy, M., Mevada, S.T., Alrawas, A... (2017). Vincristine-induced neuropathy in pediatric patients with acute lymphoblastic leukemia in Oman: Frequent autonomic and more severe cranial nerve involvement. Pediatr Blood Cancer 64(12). 
7. Baytan, B., Evim, M.S., Guler, S., Gunes, A.M., \& Okan, M. (2015). Acute Central Nervous System Complications in Pediatric Acute Lymphoblastic Leukemia. Pediatr Neurol 53(4):312-8
8. Zawitkowska, J., Lejman, M., Zaucha-Prazmo, A., Drabko, K., Plonowski, M., \& Bulsa, J...(2019). Grade 3 and 4 Toxicity Profiles During Therapy of Childhood Acute Lymphoblastic Leukemia. In Vivo, 33(4):1333-9. 